\title{
Diagnóstico Patológico en Mora de Castilla Rubus glaucus Bentham (Rosales:Rosaceae)
}

\author{
A pathological diagnosis of Rubus glaucus Bentham Andean \\ blackberry (Rosales:Rosaceae)
}

\section{Diagnóstico patológico em amora preta de Castilla Rubus glaucus Bentham (Rosales:Rosaceae)}

\author{
María Alexandra Mora-Ramos ${ }^{1}$, Ing. Agro; Fidela Patricia Pardo-Carrasco ${ }^{1}$, \\ Ing. Agr, Esp; Harold Bastidas-López ${ }^{1}$, Ing. Agr, MSc \\ 1 Universidad de los Llanos, Facultad de Ciencias Agropecuarias y Recursos Naturales, Villavicencio - Meta, Colombia.
Email: fpardoc@unillanos.edu.co
}

Recibido: 13 de febrero de 2018

Aceptado: 29 de febrero de 2020

\begin{abstract}
Resumen
Se realizó un diagnóstico patológico de un cultivo de Mora de Castilla en la vereda Pascote, municipio de Gutiérrez Cundinamarca, Colombia. Las muestras vegetales se tomaron de las hojas, tallo, flor, fruto y raíz; las cuales, se procesaron en identificaron en el laboratorio de fitopatología de la Universidad de los Llanos. En el campo se evaluó incidencia de cada síntoma. Se encontraron cuatro patógenos fungosos Colletrotrichum . gloesporoides (Penz.) Penz. con 40.00\% de incidencia, Peronospora sparsa Berk con una incidencia del 20.00 \%, Oidium sp, con $12.50 \%$ de incidencia y Botrytis cinérea Perms., con una incidencia del $7.50 \%$.
\end{abstract}

Palabras clave: diagnostico, mora de castilla, patógeno, enfermedades.

\begin{abstract}
A pathological diagnosis was made of an Andean blackberry crop (Rubus glaucus Bentham) in the rural area of Pascote, in the municipality of Gutiérrez, Cundinamarca, Colombia. Samples were taken from blackberry leaves, stems, flowers, fruit and roots; they were processed and identified in the Universidad de Los Llanos' plant pathology laboratory. Pathogen/ disease symptom incidence was evaluated in the field. Four fungal pathogens were found and identified: Colletotrichum gloesporoides (Penz.) Penz (anthracnose), 40\% incidence, Peronospora sparsa Berk (downy mildew), 20\% incidence, Oidium sp. (powdery mildew), 12.5\% incidence, and Botrytis cinerea Perms (gray mold disease), $7.5 \%$ incidence.
\end{abstract}

Como Citar (Norma Vancouver):

Mora-Ramos MA, Pardo-Carrasco FP, Bastidas-López H. Diagnóstico Patológico en Mora de Castilla Rubus glaucus Bentham (Rosales: Rosaceae). Orinoquia, 2020;24(2): 27-32. DOI: https://doi.org/10.22579/20112629.618 
Keywords: pathological diagnosis, blackberry, pathogen, diseases.

\begin{abstract}
Resumo
Realizou-se um diagnóstico fitopatológico num cultivo de amora preta no Vilarejo de Pascote, município de Gutiérrez, Cundinamarca, Colômbia. As amostras vegetais foram colhidas das folhas, caule, flor, fruto e raiz; que foram processados e identificados no laboratório de patologia vegetal da Universidad de los Llanos. A incidência de cada sintoma foi avaliada no campo. Foram encontrados quatro patógenos fúngicos: Colletrotrichum. gloesporoides (Penz.) Penz. com uma incidência de 40,00\%; Peronospora sparsa Berk com uma incidência de 20,00\%; Oidium sp, com uma incidência de 12,50\%; e Botrytis cinérea Perms., com uma incidência de 7,50\%.
\end{abstract}

Palavras-chave: Diagnóstico patológico, Amora preta, Patógeno, doenças.

\section{Introducción}

La Mora de Castilla es un cultivo herbáceo anual, según CCB 2015 es originaria de las zonas altas tropicales de América, los cuales incluyen Colombia, Ecuador, México Panamá, Guatemala, Honduras, y Salvador. La mora pertenece a la familia Rosáceae. Es una fruta muy apetecida por su sabor y variedad de usos, además de sus propiedades nutricionales, medicinales y culturales, su cultivo ha tenido un crecimiento en los últimos años en Colombia. Para Ministerio de Agricultura (2020) el área sembrada con mora en Colombia en el año 2018 fue de 15.696 Hectáreas, el área cosechada de 13.585 Hectáreas con una producción aproximada de 129.977 Toneladas. Se reporta que los Departamento en Colombia con mayor área sembrada son Cundinamarca, Santander, Boyacá y Huila, con un promedio de rendimiento de 7.99 Toneladas/Ha (Min Agricultura 2020). Uno de los limitantes de la producción de este cultivo son los problemas fitosanitarios, por este motivo este trabajo pretende identificar las enfermedades más importantes en cultivos en la vereda de Pascote en el municipio de Gutiérrez en Cundinamarca. Este trabajo es la fase inicial para determinar planes de manejo integrado de enfermedades y no depender de las aplicaciones calendario o el uso excesivo de pesticidas.

\section{Revisión de literatura}

El género Rubus es uno de los más diversos en términos morfológicos y genéticos, presentando un amplio espectro de especies silvestres y cultivadas que son apetecidas por sus frutos comestibles. En Ecuador, el cultivo de mora ( $R$. glaucus y Rubus sp.) está presente a lo largo del callejón interandino, entre 2000 y 3100 msnm. Este frutal es un rubro de importancia económica por su alta demanda para consumo en fresco y procesado. (Iza y otros 2020)

\section{Taxonomía}

Pertenece al Reino: Plantae, Subreino: Tracheophyta, Super división: Spermatophyta, División: Magno- liophyta Clase: Magnoliopsida, Subclase: Rosidae, Orden: Rosales, Familia: Rosaceae, Subfamilia: Rosoideae, Género: Rubus L, Subgénero: Lampobatus, Especie: Rubus glaucus Benth. (USDA, 2017)

\section{Morfología}

Bernal y otros 2020, sostienen que la mora de castilla en su forma natural es una planta de hábito perenne, de porte arbustivo, semierecto y semitrepador, y de crecimiento muy vigoroso; puede llegar a tener hasta tres metros de altura.

El ICA 2011, sostiene que las hojas de la mora son elípticas, oblongas enteras, puntiagudas, de color verde opaco en el envés y más claro y brillante en el haz, dispuestas en forma alterna, con bordes enteros o discretamente dentados. El Tallo es herbáceo, recto y se ramifica en secundarios y terciarios

López y otros 2019, sostienen que las flores de mora de castilla son completas y perfectas y tienen una estructura actinomórfica regular y perianto, con 5 pétalos y 5 sépalos diferenciados (heteroclorámicos). La corola tenía pétalos y sépalos libres dialipetal y dialisepal, respectivamente

Beltrán 2015, citando a Romoleroux y Nllgaard, 1996), sostienen que el fruto de la mora de castilla es una polidrupa, con 70-100 drupeolas por receptáculo (y dentro de cada una hay una semilla; los frutos pueden ser de tamaño grande, mediano o pequeño; circular o cónico elíptico, la maduración es dispareja porque la floración no es homogénea

\section{Condiciones agroclimáticas}

La mora de castilla requiere las siguientes condiciones agroclimáticas: Altura sobre el nivel del mar: 1.800 a los 2.400 m.s.n.m. Temperatura: entre 11 y $18{ }^{\circ} \mathrm{C}$. Humedad relativa: 70 al $80 \%$. Requerimiento Hídrico: precipitaciones entre 1.500 y 2.500 mm al año. Tipo de Suelo: franco arcilloso. Rango de $\mathrm{pH}$ : entre 5,2 y 
6,7 , alta susceptibilidad a las heladas y al exceso de agua, un óptimo desarrollo en suelos franco arcillosos (CCB, 2015)

\section{Enfermedades}

\section{Pudrición del fruto o moho gris (Botritys cinerea Pers. ex. Fr.)}

Una de las enfermedades que ocasiona grandes pérdidas económicas y es considerada como uno de los principales problemas fitosanitarios es la pudrición del fruto, moho gris o pudrición blanda, ocasionado por el hongo Botrytis scinerea (Quinatoa 2015)

Los frutos pueden ser afectados en todos los estados de desarrollo y presentar decoloración, pudrición, deshidratación, crecimiento de un moho de aspecto aterciopelado, de color gris, verde oliva o café claro que cubre parcial o totalmente la superficie del fruto; algunos frutos se caen, otros se momifican y permanecen adheridos a los racimos. El hongo puede avanzar hacia los pedúnculos que sostienen los frutos, donde causa pudrición seca de color café. (Saldarriaga y otros 2020).

Acosta y otros 2020, sostienen que Botritis afecta a la mora de castilla con la reducción del rendimiento, necrosis del fruto, momificación, quemaduras de las inflorescencias y pudrición del fruto

\section{Antracnosis del fruto, muerte descendente o secadera Colletotrichum gloesporioides (Penz.) Penz. y Sacc.}

Es una de las enfermedades más frecuentes y de mayor incidencia en los cultivos de frutales ubicados tanto en los climas fríos como en los cálidos (Forero de la Rotta, 2001).

En los tallos y ramas se observan manchas ovaladas de color morado o violáceo, que los cubren parcial o totalmente. Luego las lesiones se tornan negras y se secan; de ahí que en algunas zonas la enfermedad se conozca con el nombre de palo negro (Tamayo, 2003). CCB (2015) sostiene que la antracnosis ocasiona manchas oscuras en ramas y tallos que pueden llegar a producir muerte de tejido vegetal como en ramas o la planta misma; la presencia de la enfermedad es favorecida por humedades relativas altas, presencia de malezas y problemas de aireación en el cultivo.

\section{Mildeo polvoso, cenicilla o crespera Oidium sp.}

Descripción: Este es un hongo que cambia el color de las hojas y las deforma. Se localiza en ramas jóvenes, en tallos, botones y frutos; en estos tres últimos se presenta un polvillo de color blanco (CCB,2015).

\section{Mildeo velloso Peronospora sparsa Berk}

En las hojas, los síntomas no son tan frecuentes ni visibles y sólo se observan las estructuras del patógeno en el envés de las mismas (Tamayo, 2003). Los botones florales afectados presentan lesiones de color café claro a negro, que progresan hasta cubrir completamente la estructura, los pedúnculos se secan, y junto con los peciolos que acompañan la estructura floral presentan lesiones con tonalidades moradas sobre las cuales puede aparecer también la esporulación del patógeno. (Saldarriaga y otros 2020). El mildeo velloso ocasiona en frutos agrietamientos y deformaciones; ocasiona enrojecimiento prematuro en los frutos inmaduros infectados, deteriorando su calidad, incrementa especialmente en épocas lluviosas. (ICA, 2011)

\section{Metodología}

\section{Materiales requeridos para el diagnostico}

Muestra vegetales (hojas, tallo y raíz) de mora de castilla.

PDA (Papa Dextrosa Agar)

Cubetas (cámara húmeda)

Papel absorbente (cámara húmeda)

Microscopio

Porta objeto

Cinta trasparente

Lugol

\section{Trabajo en campo}

Se colectaron las muestras vegetales de mora de castilla en el sector la Hoya de la vereda Pascote del municipio de Gutiérrez Cundinamarca, a 2219 m.s.n.m., registra una temperatura media de $15^{\circ} \mathrm{C}$. Se recolectaron muestras en estado vegetativo de producción, floración y fase vegetativa.

Se tomaron muestras de hojas, tallos, frutos y flores, se rotularon para posteriormente llevarlas al laboratorio de fitopatología de la Universidad de los Llanos en Villavicencio, Meta. Esta actividad se realizó 4 veces durante el ciclo del cultivo de la mora de castilla.

\section{Trabajo en laboratorio}

Previamente se había preparado el medio de cultivo (PDA) para sembrar las muestras. En diferentes cajas Petri, se sembró una parte afectada de los órganos re- 
colectados. También se realizaron cámara húmedas, que consiste en una caja plástica con papel absorbente, humedecido con agua esterilizada hasta alcanzar máxima capacidad. Se siguió la metodología de trabajo propia del laboratorio, una guía para cámara húmeda y siembra en PDA.

Se realizaron improntas y con ayuda del microscopio se determinaron las estructuras de los hongos presentes en las muestras.

\section{Resultados y discusión}

El diagnóstico efectuado para el cultivo de Mora de Castilla, presento la presencia de enfermedades de tipo fúngico como: Antracnosis caudada por Colletotrichum gloesporoides (Penz.) Penz. y Sacc „, con 40\% de incidencia, Oidium sp,con $12.50 \%$ de incidencia, Peronospora sparsa Berk con una incidencia del 20.00 $\%$ y Botrytis cinérea Perms., con $7.50 \%$ de incidencia, siendo algunos de estos patógenos similares a los encontrados por Hernández 2004 y otros en la zona de la Vega Cundinamarca en mora de castilla, encontraron una incidencia del 34,8\% de Collectotrichum; $18 \%$ de Alternaria, y $15,8 \%$ de Oidium. También se observaron patógenos como Peronospora, Botrytis y Phoma,

El C. gloesporoides se observó en hojas, tallos, flores y frutos en todos los estados fisiológicos, similar a lo reportado CCB 2015, Saldarriaga y otros 2020.
El mildeo Velloso causado por P. sparsa se observó en las hojas especialmente en todos los estados fenologicos pero también se reporta en tallos y algunos frutos, similar a lo reportado por ICA 2011 quien afirma que es una enfermedad que afecta tallos, hojas y frutos, estos presentan maduración desigual. pérdida de turgencia.

Por otra parte, los síntomas característicos de Cenilla (Oidium sp.), fueron hallados en la plantación con una baja frecuencia, sobre todo en hojas jóvenes observando algunas malformaciones, similar a lo encontrado por Saldarriaga y otros 2020, el cual dice que el mildeo polvoso afecta principalmente a las hojas jóvenes, el síntoma más común es la deformación, enroscamiento o encrespamiento de las hojas con presencia de manchas cloróticas irregulares y difusas.

Se encontró también Botritis cinérea el nominado el moho gris en botones florales y en frutos en todos los estados de fructificación. Forero de la Rota 2007 sostiene que $B$. cinerea infecta los botones florales de la mora y permanece quiescente hasta el momento de la fructificación y maduración, cuando la enfermedad se manifiesta y llega a ocasionar pérdidas superiores al $50 \%$.

En la Figura 1, se detallan los resultados de laboratorio y los estados de afección del cultivo de Mora de Castilla por enfermedades y el desarrollo del hongo en laboratorio.

Tabla 1. Enfermedades diagnosticadas en el laboratorio de la Universidad de los Llanos.

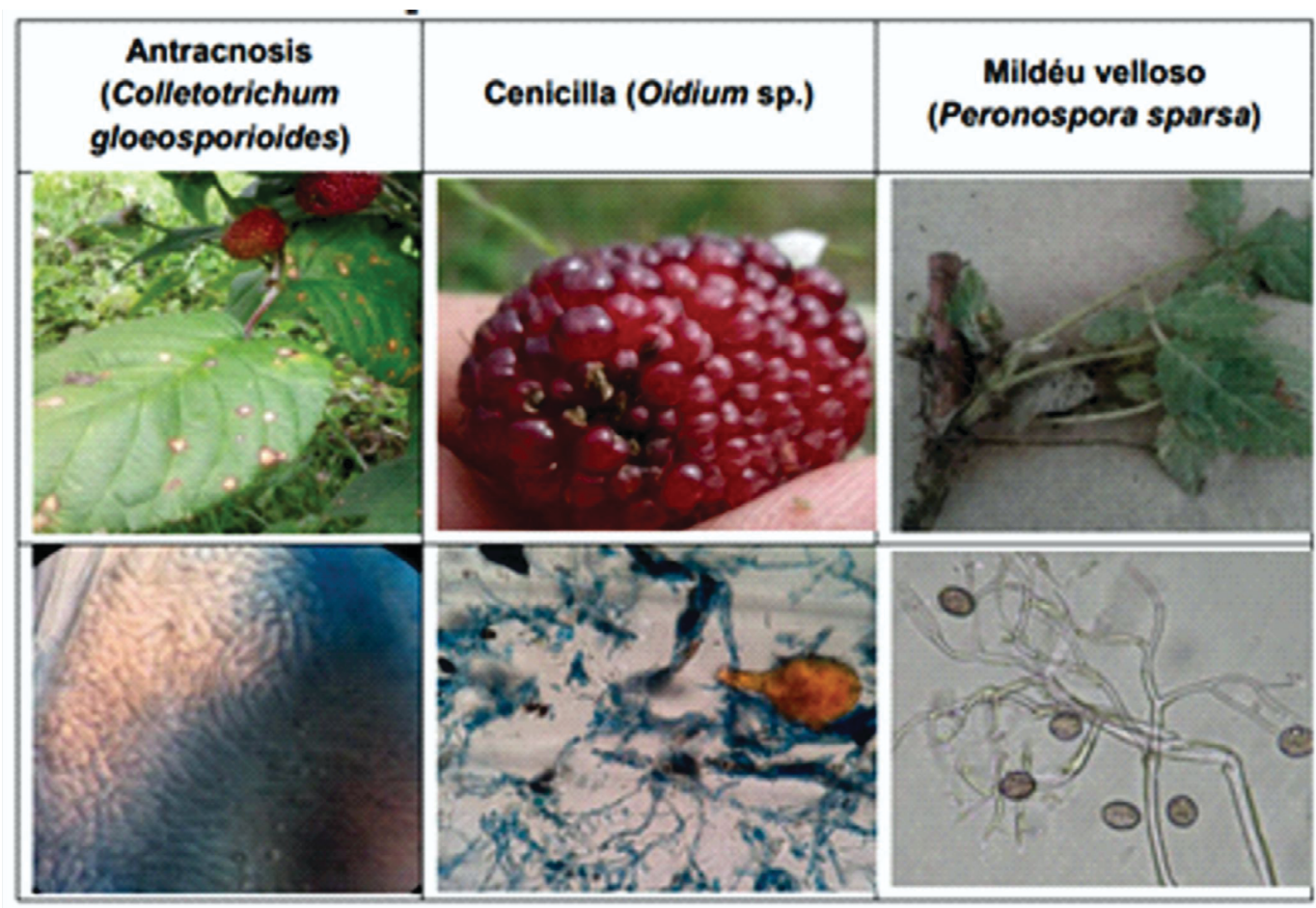



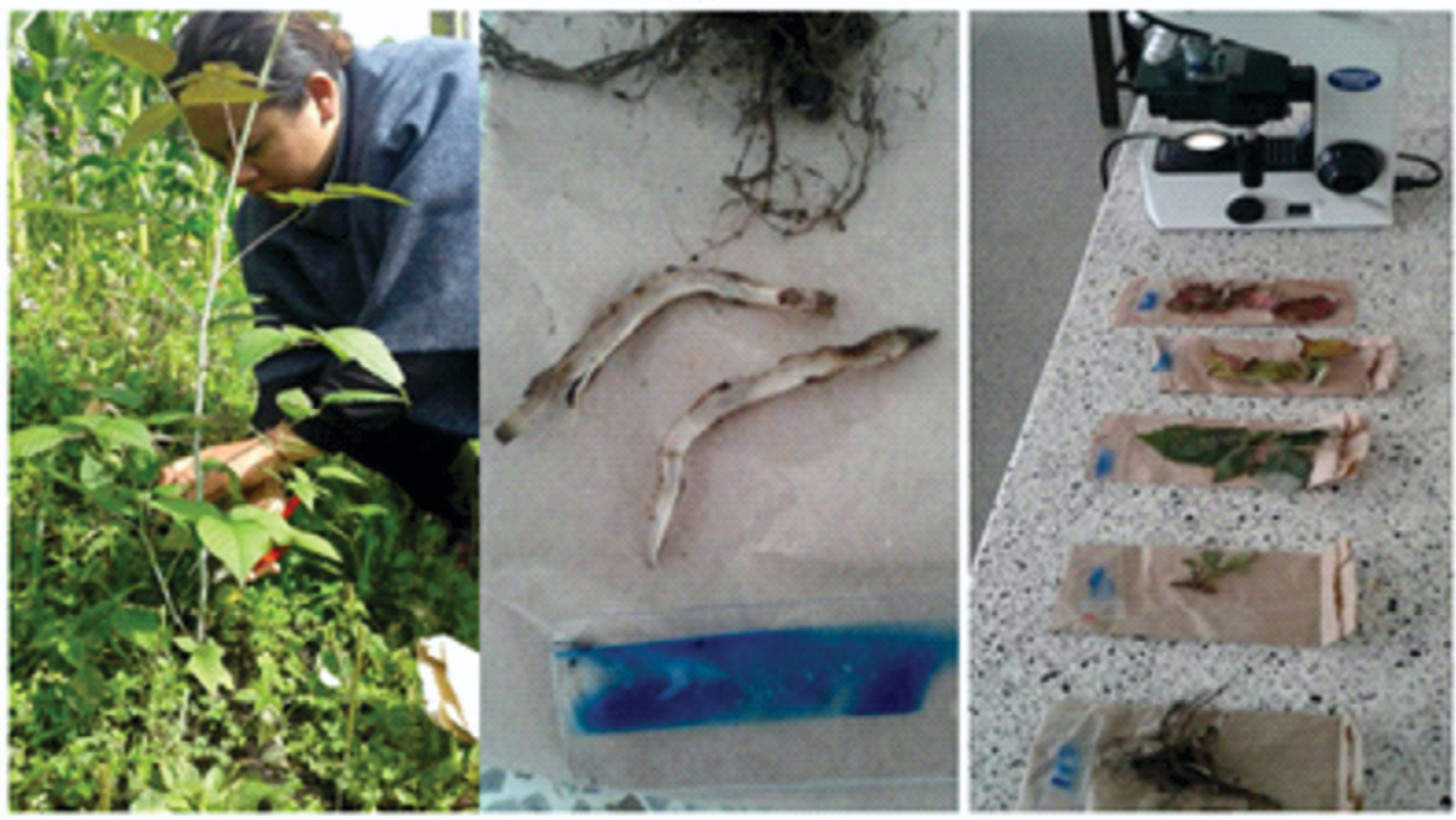

Figura 1. Las imágenes de izquierda a derecha representan: toma de muestras vegetales, corte de raíz, diagnóstico de laboratorio.

Se encontraron cuatro patógenos fungosos C. gloesporoides (Penz.) Penz. con $40.00 \%$ de incidencia, Peronospora sparsa Berk con una incidencia del $20.00 \%$, Oidium sp, con $12.50 \%$ de incidencia y Botrytis cinérea Perms., con una incidencia del $7.50 \%$.Este tipo de reconocimiento de patógenos se debe realizar en la zonas frías del Departamento del Meta y Cundinamarca, en este cultivo y en otros cultivos que no se les da la importancia que se le debe dar.

\section{Referencias}

Acosta M, Viera WF, Jackson T, Vásquez W. Alternativas tecnológicas para el control de Botrytis sp. en mora de Castilla Rubus glaucus UTE. Quito Ecuador. 2020;11(2):Marzo-junio

Beltrán E. 2015. Caracterización fisiológica de Rubus glaucus en función de crecimiento productivo y de microambiente en el corregimiento del Ecano, Nariño. Tesis de Biólogo. Facultad de Ciencias exactas y naturales Universidad de Nariño. Pasto. 153 $\mathrm{p}$

Bernal J, Díaz C, Franco G. 2020. Generalidades de la Mora de Castilla. En: Tecnología para el cultivo de la mora Rubus glaucus Beth. Agrosavia. Mosquera Colombia. Pp. 39-139

CCB. Cámara de comercio de Bogotá. 2015. Manual Mora. Programa de apoyo agrícola y agroindustrial de la vicepresidencia. Fortalecimiento empresarial de la Cámara de Comercio de Bogotá. Gills y Saens. Bogotá Colombia. 54p

Forero de La Rota, Muñoz V. 2001. Enfermedades de la mora de castilla. Boletín de Sanidad Vegetal Nº 34 Instituto Colombiano Agropecuario ICA. Produmedios. Bogotá. 36 pp.
Forero de La Rotta MC. 1993. Enfermedades de importancia económica en mora de castilla (Rubus glaucus Benth). En XIV congreso de la asociación colombiana de fitopatología y ciencias afines. Resúmenes de trabajos. Santa Marta.

Forero de La Rotta MC. 2007. Uchuva (Physalis peruviana L.) y mora de Castilla (Rubus glaucus Benth.): enfermedades y manejo. En Memorias del XXVIII Congreso de la Asociación Colombiana de Fitopatología y Ciencias Afines. Palmira, Valle del Cauca, Colombia: Asociación colombiana de fitopatología y ciencias afines (Ascolfi).

Hernández D, Árdila S, Díaz J, Perilla A, Cubillos D, Serrano J, et al. Caracterización de agentes causales de enfermedades en el cultivo de mora (Rubus glaucus) en la finca manantial en la vereda sabaneta, municipio de La Vega, Cundinamarca. Revista de Ciencias Agropecuarias Universidad de Cundinamarca 2014;4(1):9-17.

ICA. 2011. Manejo Fitosanitario del cultivo de la Mora de Castilla Rubus glaucus. ICA, Instituto Colombiano Agropecuario. Produmedios. Bogotá Colombia. P 31

Iza M, Viteri P, Hinojosa M, Martínez A, Sotomayor A, Viera W. Diferenciación morfológica, fenológica y pomológica de cultivares comerciales de mora (Rubus glaucus Benth.). Enfoque UTE, 2020;11(2):47-57.

López AA, Marulanda ML, Gómez LM, Barrera C. Rubus glaucus Benth: morfología y biología floral dirigida a procesos de mejoramiento de plantas. Rev Fac Nac Agron, Medellín. 2019;72(3):8909-8915.

Motoya CA, Florez UV, Hincapié E. 1997. Principales enfermedades y plagas en el cultivo de la mora. Boletín Técnico Instituto Colombiano Agropecuario ICA- Unidad Municipal de Asistencia 
Técnica Agropecuaria Umata-Quinchía. Litógrafos Asociados Ltda. "Litoas"

Ministerio de Agricultura de Colombia 2020. Presentación Subsector productivo de la mora .Dirección de Cadenas Agrícolas y Forestales Grupo de Cultivos Permanentes y Hortifrutícolas Secretaría Técnica Nacional de las Cadenas de Frutos Rojos

Romoleroux K, Nllgaard B. 1996. Flora of Ecuador: Rosaceae ; Connaraceae. Department of Systematic Botany, University of Goteborg.

Quinatoa N. 2015. Evaluación del control de botrytis (Botrytis cinerea) en el cultivo de mora (Rubus glaucus Benth) mediante el uso de Trichoderma y EMAS en la comunidad de Misquillí de la parroquía Santa Rosa, provincia de Tungurahua. Ambato, Ecuador: Universidad Técnica de Ambato.

Saldarriaga A, Betancourt M, Tamayo P, Martinez E, Zapata Y, Beltran C, Cotes A. 2020. Enfermedades asociadas a la mora de castilla. En: Tecnología para el cultivo de la mora Rubus glaucus Beth. Agrosavia Colombia. Mosquera Colombia Pp. 221-256.

United States Department of Agriculture (usda). 2017. Rubus glaucus Benth. Andes Berry Classification. In Plant Data Base. Natural Resources Conservation Service. https://plants.usda.gov/ core/profile?symbol= RUGL5.

Maria Mora: https://orcid.org/0000-0003-4368-6957 Fidela Pardo: https://orcid.org/0000-0002-1535-7534 Harold Bastidas: https://orcid.org/0000-0002-6544-1224 